\title{
Robust Airborne Ultrasonic Positioning of Moving Targets in Weak Signal Coverage Areas
}

\author{
Joaquín Aparicio, Member, IEEE, Teodoro Aguilera, and Fernando J. Álvarez, Senior Member, IEEE
}

\begin{abstract}
Current ultrasonic Local Positioning Systems (LPS) based on an infrastructure of beacons can provide centimeter-level accuracy employing the spread spectrum technique, which also adds robustness against noise. However, the strong attenuation of the acoustic waves at high frequencies, the high directionality of ultrasound transducers, and the Doppler effect caused by moving targets still affect the correct performance of LPS. These phenomena reduce the availability of these systems in weak signal coverage areas, as they are no longer able to distinguish weak arrivals from spurious peaks, failing to calculate the position of the target. In this work, the aforementioned problems are dealt with by transmitting Doppler resilient waveforms together with a validation code based on Complementary Set of Sequences. This validation code is leveraged at the receiver after Doppler compensation to reduce the number of spurious arrival candidates and therefore increase the system availability. Compared to a system with no validation, experimental tests with a moving robot have shown that the proposed system increased the availability in weak coverage areas between 20 and $25 \%$. The robot's average 2D positioning error at rest and in motion was $4.6 \mathrm{~cm}$ and $6 \mathrm{~cm}$, respectively.
\end{abstract}

Index Terms-Doppler, Indoor Positioning, OFDM, Ultrasonic.

\section{INTRODUCTION}

$\mathbf{L}$ OCAL positioning systems (LPS) have been a subject of intense research during the last three decades. With a limited applicability at the beginning, mainly conditioned by the need to use specifically designed receivers, the development of these systems is boosted during the first years of this century by the mass commercialization of smart portable devices, and the consequent appearance of an increasing number of Location-Based Services (LBS) [1].

Today, a variety of technologies coexist that satisfy different requirements of coverage, precision and cost [2], thus finding application in a diversity of fields [3]. Among these technologies, ultrasonic systems are considered a classical and reliable solution, which are characterized by a centimeter precision with coverage ranges of up to some tens of meters [4], [5]. This relatively high precision is provided by the incorporation of broadband signaling and the pulse compression detection technique, which also ensure a high robustness to in-band noise. The high performance of current ultrasonic LPS is fostering

This work has been supported in part by the Spanish Government and the European Regional Development Fund (ERDF) through Project MICROCEBUS under Grant RTI2018-095168-B-C54, in part by the Regional Government of Extremadura and ERDF-ESF under Project GR18038, and in part by the Research Council of Norway, under project 269614/O20: "Infrastructure-less indoor positioning in 3D".

J. Aparicio is with the Department of Informatics, University of Oslo, Oslo, 0373 Norway (e-mail: joaqapar@ifi.uio.no).

T. Aguilera and F. J. Álvarez are with the Department of Electrical Engineering, Electronic and Automation, University of Extremadura, Badajoz, 06006 Spain (e-mail: teoaguibe@unex.es; fafranco@unex.es). the development of new and more demanding applications, such as the detection and tracking of fast-moving vehicles [6]-[8], an ambitious challenge that must face new design problems.

One of these problems is the Doppler effect, which has a large influence on airborne acoustic systems due to the relatively low propagation speed of their sensing signal $(\approx 343 \mathrm{~m} / \mathrm{s}$ at $20^{\circ} \mathrm{C}$ ). Some authors have already analyzed the deterioration that this effect can cause on the matched filtering detection of the broadband signals emitted by an ultrasonic LPS [9], [10], and some works can also be found in the literature that propose partially Doppler-tolerant designs [11]-[13]. In a more recent work [14], the authors have presented a new Doppler compensation algorithm that, using signals based on the concatenation of a chirp with a carrier pulse, is capable to calculate the time shift suffered by the compressed detection pulse, and thus accurately estimate the position of a fastmoving target.

An additional problem that has not deserved much attention from the ultrasonic LPS community is the operation in weak signal coverage areas. Only a few works address the problem of low coverage in ultrasonic LPS [15], [16]. However, these works do not provide solutions to improve the performance of the system in poor signal coverage regions, but they only propose to increase the size of the coverage areas. The ultrasonic LPS coverage problem is a direct consequence of both the typically narrow beamwidth of high-frequency acoustic transducers [17] and the large attenuation that these mechanical signals undergo in the air [18]. Most works use a detection 
threshold below which no signal is validated, which must be high enough as to avoid the detection of spurious peaks. In weak signal coverage areas some of the actual arrival peaks may not be detected, and no position is usually computed since the set of positioning equations is therefore undetermined. In this case, the last valid position or a prediction based on the evolution of the last valid positions can be rendered by the system. An alternative approach consists in discarding the use of any threshold and always assume the detection of a received signal. Under weak coverage, false detections may be validated leading to erroneous computations of the position or even the divergence of the localization algorithm. These outliers are typically discarded using statistical analysis on a set of measurements taken in similar conditions.

None of the approaches mentioned above is a valid solution when tracking fast-moving targets for obvious reasons. This work proposes a complex signal coding scheme based on the use of an Orthogonal Frequency Division Multiplexing (OFDM) modulated Complementary Set of Sequences, which is appended to the Doppler tolerant header presented in [14]. In this case, the combined chirp + carrier header is used to correctly baseband demodulate the appended tail and thus validate the detection of weak signals. This is, to the authors knowledge, the first work that proposes such a strategy to validate the actual reception of weak signals in an airborne ultrasonic LPS and avoid the computation of erroneous positions.

The rest of the paper is organized as follows. Section II presents a comprehensive review of directly related works. In Section III the emitter and receiver modules, and the signal coding scheme of the proposed system are described in detail. Section IV presents and discusses a set of experimental results obtained in a real environment. Finally, Section V highlights the most relevant conclusions that can be drawn from this work.

\section{Related Works}

First airborne ultrasonic positioning systems emerged during the last years of the former century, based on the emission of short and constant-frequency ultrasonic pulses whose arrival was detected by following a simple energy thresholding procedure. This is the case of the Active Bat System [19] where wireless badges (bats) carried by personnel or attached to certain equipment emitted $40 \mathrm{kHz}$ ultrasonic pulses of $50 \mu$ s duration after being triggered over a wireless link. These pulses were received by a set of ceiling-mounted sensors that measured the pulses Time-of-Arrival (TOA) and computed the badge three-dimensional position by spherical lateration. A different approach was proposed in the Constellation System [20], where a set of ultrasonic emitters was deployed at known locations in the environment. These beacons emitted a $40 \mathrm{kHz}$ ultrasonic pulse after receiving an infrared trigger code from the unit to be located, which communicated with the beacons one-at-a-time. The receiving unit needed to compute at least three TOAs from the emissions of different beacons to obtain its position by spherical lateration. Note that, in this case, the device to be located was in charge of computing its own position using the signals emitted from different beacons. This architecture has been defined by some authors as privacyoriented, in contrast to the centralized architecture of the Active Bat system. An additional example of a narrowband ultrasonic LPS that can act both as centralized or privacyoriented is the Cricket system [21]. This system was based on a set of independent beacons that incorporated a Radio Frequency (RF) transceiver, an ultrasonic emitter, and an ultrasonic receiver. A target could compute its coarse position by measuring the TOAs of the $40 \mathrm{kHz}$ ultrasonic pulses of $125 \mu$ s duration emitted by nearby beacons, which also broadcast their own position through a $433 \mathrm{MHz}$ RF signal. Instead of calculating an absolute position value, the system associated the target location to that of the measured closest beacon. All these systems featured very simple emitter and receiver acoustic modules but at the expense of providing a limited positioning accuracy of some decimeters with high sensitivity to in-band noise. Moreover, special attention had to be paid to avoid interference between different emitters, either by making use of time multiplexing strategies [20] or by developing specific algorithms [21].

A solution to these limitations was soon provided by the pulse compression technique extensively used in radar systems [22]. A new generation of broadband ultrasonic LPS started being developed in the early 2000s, based on the emission of Binary Phase Coded signals that were detected by matched filtering. This spread spectrum technique had been successfully incorporated in the development of high precision airborne sonars some years before [23]-[25], so its application in the field of ultrasonic LPS was rather straightforward. One of the first broadband ultrasonic LPS was presented in [26], [27], where the authors proposed the use of 511-bit Gold codes to modulate a $50 \mathrm{kHz}$ ultrasonic carrier with a bit period of $50 \mu \mathrm{s}$, thus giving a total emission duration of $25.55 \mathrm{~ms}$. In [26], eight receivers were installed in the ceiling of an office room to configure a centralized architecture that measured the TOAs of the signals emitted by a set of synchronized transmitters. The authors reported positioning accuracies slightly above $2 \mathrm{~cm}$ when using spherical lateration in a noisy environment. In [27], a privacy-oriented architecture was presented with positioning accuracies around $5 \mathrm{~cm}$. A similar privacy-oriented architecture was presented in [28], based on the modulation of a $50-\mathrm{kHz}$ carrier with 127-bit Gold codes and a bit period of $20 \mu \mathrm{s}$, for a significantly shorter emission duration of $2.54 \mathrm{~ms}$. The main contribution of this broadband ultrasonic LPS was the proposal of a hyperbolic lateration algorithm, based on the measurement of the Time-Difference-of-Arrival (TDOA) between the first detected signal, emitted by the nearest beacon, and the other signals detected subsequently. This positioning strategy avoided the need for a synchronized triggering signal between the beacons and the receiver. An improved version of this system was presented in [29], where the $50 \mathrm{kHz}$ carrier was modulated by 255-bit Kasami codes to obtain accuracies below $1 \mathrm{~cm}$ in the horizontal positioning of the receiver. Since the appearance of these initial works, other pseudorandom sequences have been proposed to encode the emissions of more computationally efficient broadband ultrasonic LPS, such as Loosely Synchronous Sequences [30], 
Complementary Sets of Sequences [31] or Zadoff-Chu Codes [32]. In all these broadband systems, the TOA or TDOA of the received signal was measured when the auto-correlation peak of this signal exceeded a detection threshold, which improved the precision of the range measurement between one and two orders of magnitude with respect to that of the previous narrowband systems. Nevertheless, the longer duration of these signals, as well as the capability to simultaneously emit them following a Code Division Multiple Access (CDMA) method, magnified the problems derived from multipath propagation. Some authors have mitigated this problem by using an OFDM modulation scheme [33], estimating the line-of-sight TOA with a Matching Pursuit algorithm [34], or introducing a validation method based on the repetition of the same code [35].

Regardless of the new inconveniences derived from multipath propagation, the improved performance of broadband ultrasonic LPS has recently encouraged researchers to develop new systems for the location and tracking of fast-moving targets [6]-[8]. The main problem that these researchers have to face is, as stated before, the effect that the Doppler shift has in the matched filtering detection of the received signals. To cope with this problem, in [12] the authors presented an active system based on a CDMA architecture, where an $\mathrm{m}$-sequence was emitted jointly with a carrier pulse to determine the Doppler shift. Subsequently, a resampled replica of the received signal was generated according to the suffered frequency displacement. In a different work [11], a receiver composed of a set of seven banks of five Kasami correlators was proposed, each one matched to a different frequencyshifted version of the codes to be detected, also providing a coarse estimation of the receiver's radial speed. A different approach, based on a Differential Binary Phase Shift Keying (DBPSK) modulation scheme, was explored in [36]. This technique provided better performance than the classical correlation with Binary Phase Shift Keying (BPSK) modulation for moving objects, although this performance slightly worsened for stationary objects. In [14], two of the authors of this work have recently presented a new Doppler compensation algorithm that, using signals based on the concatenation of a chirp with a carrier pulse, is capable to calculate the time shift suffered by the compressed detection pulse and accurately estimate the position of a fast-moving target.

\section{SyStem DEscription}

This section describes the ultrasonic LPS proposed in this work. The system has been installed in a laboratory environment where a positioning area of $2.4 \mathrm{~m} \times 2.4 \mathrm{~m}$ has been defined. The proposed assembly consists of an emitter and a receiver module, which are detailed below, together with the design of the transmitted acoustic signals.

\section{A. Emitter Module}

The emitter module is composed of a dedicated board based on the NXP LPC1768 microcontroller [37], which includes an 8-bit resolution Digital-to-Analog Converter (DAC), and a signal conditioning stage to drive sequentially a set of five ultrasonic beacons Prowave 328ST/R160 [38]. Moreover, the beacons' location has been chosen to minimize the Geometric Dilution of Precision (GDOP) [39]. Each beacon positions have been determined using a laser rangefinder [40] that provides $\pm 1 \mathrm{~mm}$ of accuracy. Finally, the whole module is powered from the mains via an AC/DC converter.

Fig. 1a shows an illustration of the emitter module, where its internal components, as well as the ultrasonic beacons and their spatial distribution, can be observed.

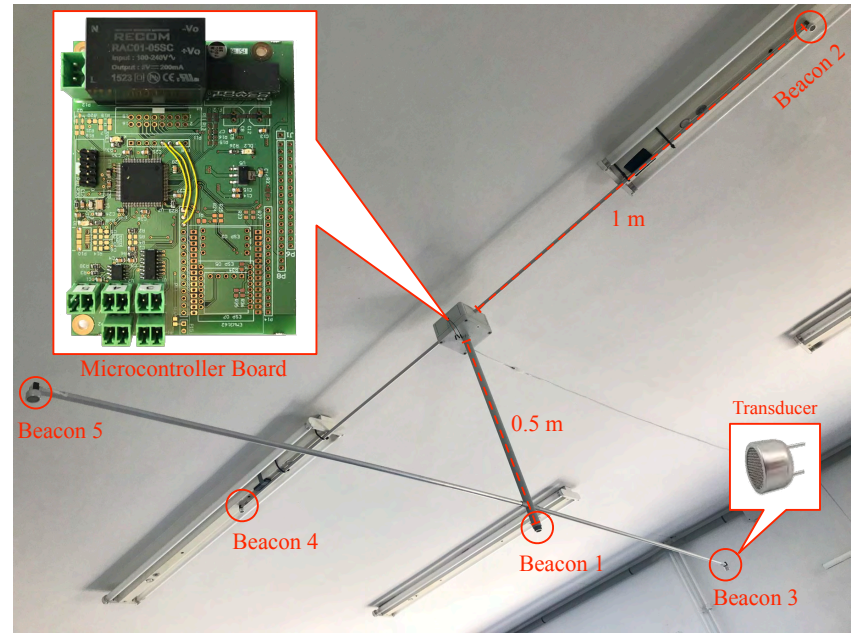

(a) Components and beacons distribution.

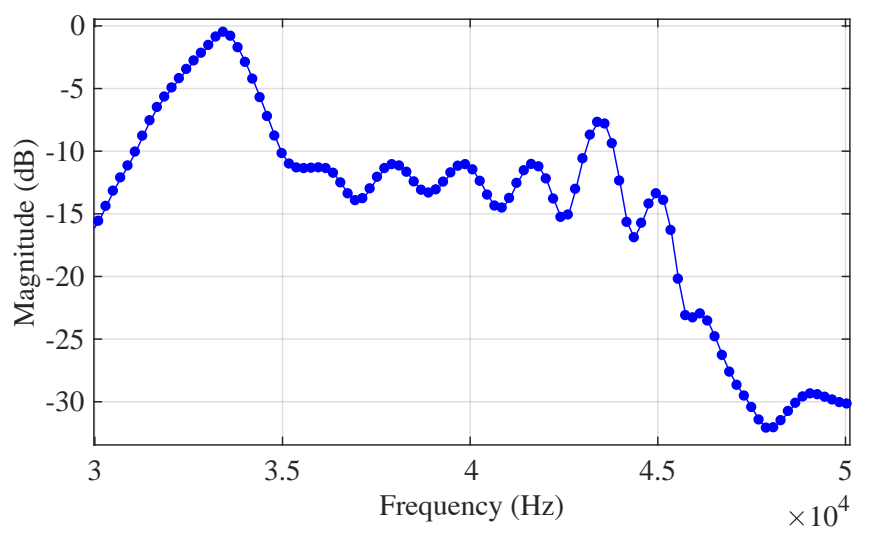

(b) Transducer frequency response.

Fig. 1: Emitter module description.

The transducer frequency response shown in Fig. 1b has been obtained by placing the emitter one meter away from the receiver [41] and aligning their respective acoustic axes. First, a preliminary frequency sweep has been performed by emitting carriers from 30 to $50 \mathrm{kHz}$. After the obtained amplitudes analysis, the optimal transducer working range has been established between 35 and $45 \mathrm{kHz}$. Subsequently, a second more exhaustive frequency sweep has been carried out where the emitted carriers amplitude has been measured every tenth of $\mathrm{kHz}$ within the range of interest. Finally, the acoustic power emitted at a one-meter distance to the source was also measured, obtaining a value of $35.4 \mathrm{~dB}$.

\section{B. Modulation and codification}

This subsection describes in detail the design of the acoustic signals that are transmitted by the beacons. A similar design 
was employed by one of the authors in an underwater communication system [42], in order to correctly detect packets of data under strong impulsive noise conditions. In this work however, the main benefit drawn from this design will be to increase the availability of the system, effectively increasing the coverage area of the LPS. The benefits of this signal design will be obtained by performing a two-step detection and validation process at the receiver, which will be described in section III-C.

Each beacon transmits a signal with three components: 1) a chirp to detect candidate arrivals, 2) a carrier to calculate the Doppler shift, and 3) a code, different for each beacon, that will allow to validate or discard these candidate arrivals. The chirp has a duration of $10 \mathrm{~ms}$, and it is transmitted with a linear increase of frequencies between 36 and $44 \mathrm{kHz}$. The reason to use chirps to provide candidate arrivals is because they exhibit better resilience against the Doppler effect than spreadspectrum signals: when the receiver is moving, the correlation peak will slightly shift in time, but its amplitude remains approximately constant, whereas the correlation peak is greatly affected by the Doppler effect [43]. Since the ultrasonic LPS drives the beacons sequentially, the same chirp will be used in all beacons, since beacon identification will be obtained later with the code in the validation step. Next to the chirp, a $10 \mathrm{~ms}$ carrier is transmitted at $40 \mathrm{kHz}$ in order to calculate the Doppler effect experienced by the signal.

The last component of the transmitted signals consists of a modulated code, placed after the carrier. As mentioned before, this code will validate or discard a candidate arrival provided by a chirp detection, and if validated, will also identify the transmitter beacon. In this work, Complementary Sets of Sequences (CSS) [44] have been selected for this purpose, due to their ideal auto- and cross-correlation properties.

A CSS consists of $M$ binary sequences in a set, where each of the $M$ sequences has length $L, M$ is a power of two, and $L$ is a power of $M$ :

$$
\begin{aligned}
M & =2^{m} \\
L & =M^{l}=2^{m \cdot l},
\end{aligned}
$$

where $m, l$ belong to the set of natural numbers, excluding zero. If the Auto-Correlation Function of a sequence $S_{i}$ of the set, $\mathrm{ACF}_{S_{i}}$, is given by:

$$
\mathrm{ACF}_{S_{i}}[\tau]=\sum_{j=1}^{L} S_{i}[j] \cdot S_{i}[j+\tau],
$$

where $i=1,2, \ldots, M$, then the Sum of the Auto-Correlation Functions (SACF) of the $M$ sequences of the set has the following property:

$$
\operatorname{SACF}[\tau]=\sum_{i=1}^{M} \operatorname{ACF}_{S_{i}}[\tau]=M \cdot L \cdot \delta[\tau],
$$

where $\delta$ is the Kronecker delta function. This way, the SACF provides a maximum of magnitude $M \cdot L$ for $\tau=0$, and a value of 0 otherwise.

With regard to the cross-correlation, for a number of sequences $M$, a group of $M_{o}$ mutually orthogonal members of
$M$-CSS of length $L$ can be found, where $M_{o} \leqslant M$. If $S_{i}^{a}$ and $S_{i}^{b}$ are the $i$-sequences of two different $M$-CSS $a$ and $b$ from an orthogonal set of size $M_{o}$, their Cross-Correlation Function is given by:

$$
\mathrm{CCF}_{S_{i}^{a} S_{i}^{b}}[\tau]=\sum_{j=1}^{L} S_{i}^{a}[j] \cdot S_{i}^{b}[j+\tau],
$$

whereas the Sum of Cross-Correlation Functions (SCCF) obeys:

$$
\operatorname{SCCF}[\tau]=\sum_{i=1}^{M} \operatorname{CCF}_{S_{i}^{a} S_{i}^{b}}[\tau]=0, \forall \tau .
$$

Which means that the SCCF of the $i$-sequences of any pair of members $a$ and $b$ from the orthogonal set provides an ideal cross-correlation. Therefore, $M_{o}$ different users can be identified after assigning a different $M$-CSS of the group to each user. When designing a peak detector, this ideal auto- and cross-correlation behavior of CSS represents a considerable advantage over other coding schemes, since the detector only has to check the value at $\tau=0$, and compare it with a pre-defined static threshold to validate or reject the candidate arrival.

The fact that the beacons transmit sequentially has been leveraged in the design by considering only $M_{o}=2$ orthogonal CSS: one to identify a reference beacon (B1), an a second one for the remaining four beacons (B2 to B5). This way, by properly identifying the reference beacon, the other validated peaks can be identified too by checking in which time slot they are received.

Considering $M=2$ and $L=32$, 2-CSS of length 32 bits will be then transmitted by the beacons, maintaining an ideal SCCF and a reasonably short duration. However, if $M_{o}=5$ orthogonal CSS were to be considered, as $M_{o} \leqslant M$, and $M=2^{m}$, then $M$ should be 8 at least, imposing also a higher value on $L$ to keep the ideal properties. Higher $M$ and $L$ results in longer sequences, which would then decrease the position update rate of the system, and would have higher position errors caused by the motion of the receiver.

The CSS codes have been modulated in OFDM due to its properties with regard to easy modulation/demodulation based on the Fourier transform, simple channel equalization in the frequency domain, some multipath resilience by using a cyclic prefix, and certain immunity to impulsive noise [45]. The bits conforming the $M$ sequences of the CSS have been mapped in Quadrature Phase-Shift Keying (QPSK), where the QPSK symbols for the user $q, x^{q}$, have been obtained by assigning one sequence of the 2-CSS to the real part of the QPSK symbol, and the other sequence to the complex part. Therefore, the $n$ symbol is given by:

$$
x^{q}[n]=S_{1}^{q}[n]+j \cdot S_{2}^{q}[n],
$$

where $S_{1}^{q}$ and $S_{2}^{q}$ are the $M=2$ sequences of the set. Thus, the 2-CSS of length 32 are mapped to 32 complex QPSK symbols, which are assigned to $K=32$ subcarriers in an $8 \mathrm{kHz}$ bandwidth between 36 and $44 \mathrm{kHz}$, obtaining a modulated signal with a duration of $4 \mathrm{~ms}$. A cyclic prefix of the same duration is added at the beginning, in order to protect the 
modulated signal against multipath arriving up to $4 \mathrm{~ms}$ after the main arrival, which is consistent with the multipath delay coming from walls around the area of interest. The duration of the OFDM-modulated CSS is then $8 \mathrm{~ms}$. Additionally, in order to equalize the channel and demodulate the CSS at the receiver, 32 pilot symbols are OFDM-modulated in the same way and using the same subcarriers and cyclic prefix duration, adding an extra $8 \mathrm{~ms}$. Therefore, the total duration of the 2-CSS of length 32 is $16 \mathrm{~ms}$, and the total duration of the transmitted signal, including the chirp and carrier, is then $36 \mathrm{~ms}$. Based on this duration, a time slot of $40 \mathrm{~ms}$ has been used in the Time-Division Multiple Access (TDMA) transmission protocol for each beacon. A diagram with the proposed emission architecture and signal design can be observed in Fig. 2.

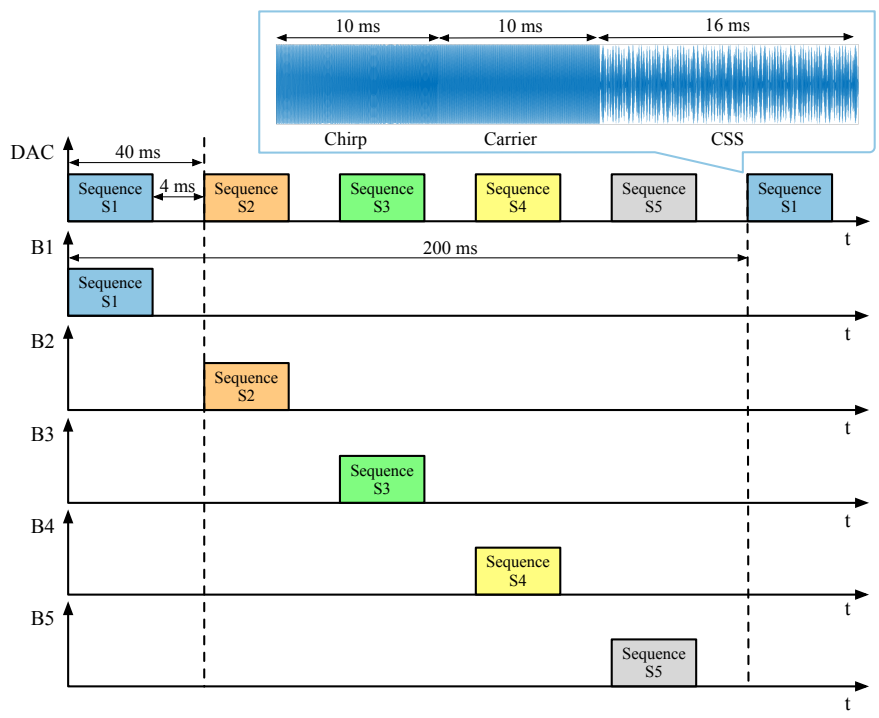

Fig. 2: Proposed emission architecture and signal design.

\section{Receiver Module}

A block diagram depicting how the receiver operates is shown in Fig. 3. A GRAS 40BE 1/4 inch prepolarized freefield microphone is continuously sampling the received signal $y(t)$ at the target, at a sampling frequency $f_{s}=500 \mathrm{kHz}$, storing the data in a laptop for later processing. The recorded data is cut into frames of $240 \mathrm{~ms}$, with an overlap of $40 \mathrm{~ms}$. As the transmission of the five beacons of the ultrasonic LPS takes $200 \mathrm{~ms}$, there will always be six receptions in one frame, where one beacon is received twice. This process ensures that, if the beacons are correctly validated, there will be enough information in one frame to calculate the location of the target with an update rate of $5 \mathrm{~Hz}$.

The samples of the received frame $y[n]$, can be expressed as a function of the channel response $h[n]$, the transmitted signal $x[n]$, and additive white Gaussian noise $z[n]$, as:

$$
y[n]=h[n] \circledast x[n]+z[n],
$$

where $\circledast$ is the convolution operator, and $n=1, \ldots, N_{f}$, being $N_{f}$ the number of samples in the received frame.

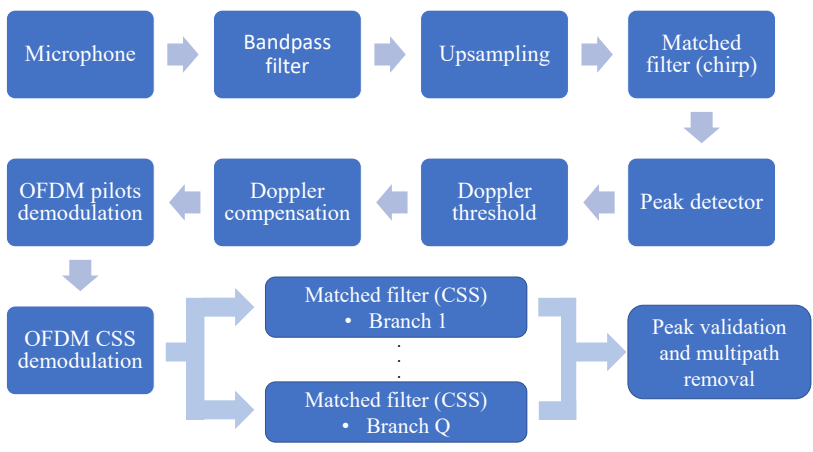

Fig. 3: Block diagram of the receiver.

Each received frame first goes through a bandpass filter to remove noise out of the frequency band of the transducer, and the frame is upsampled to $1 \mathrm{MHz}$, in order to have better resolution in the subsequent correlation operation.

The detection and validation of the arrivals is done in two steps: 1) candidate arrivals are detected, including potentially spurious ones caused by impulsive noise and multipath, in order to also detect low amplitude arrivals caused by the nearfar effect; 2) the candidate arrivals are validated or rejected, and the beacons are identified. In the first step, the $N$ samples in the upsampled data are fed to a matched filter that looks for the transmitted chirp by using a sliding window of size $N^{\prime}<N$. In this work, this window has a duration of $40 \mathrm{~ms}$ with a $10 \mathrm{~ms}$ overlap, and in each window a peak detector [46] provides the candidate arrivals. After processing all the windows in a frame, this step provides a list of $n_{c}$ candidate arrivals, indicating the first sample of each detected chirp.

The Doppler shift $D$ experienced by the received signal is evaluated next for each of the $n_{c}$ candidate arrivals, by analyzing the carrier placed after the chirp. The Doppler shift is given by:

$$
D=f_{C W}^{\prime}-f_{C W}
$$

where $f_{C W}^{\prime}$ is the frequency corresponding to the maximum of the Fourier transform of the received carrier, and $f_{C W}=$ $40 \mathrm{kHz}$, which is the center frequency of the transmitted carrier. This estimated Doppler shift can also be leveraged to remove spurious candidates, by comparing it to a threshold level $D_{t h}$, discarding those candidates in which $D>D_{t h}$. A value of $D_{t h}=1 \mathrm{kHz}$ has been used in this work, which means that for a $40 \mathrm{kHz}$ signal, the maximum speed that can be detected is approximately $8.5 \mathrm{~m} / \mathrm{s}$, much higher than the average walking speed. Thus, the set of $n_{c}$ candidate arrivals is reduced to $n_{c}^{\prime}$. For those final $n_{c}^{\prime}$ candidate arrivals, this Doppler shift is compensated by a resampling operation.

The OFDM signals corresponding to the pilot symbols and CSS, placed next to the carrier, are extracted next and downsampled back to $f_{s}=500 \mathrm{kHz}$. Demodulation of both pilots and CSS OFDM signals is analogous. Pilot symbols are demodulated first, where the received pilots $Y_{p}[k]$ are contained in the $K$ subcarriers:

$$
Y_{p}[k]=H[k] \cdot X_{p}[k],
$$


where $k=1, \ldots, K$. Since $X_{p}[k]$ are the known transmitted pilot symbols, the channel response $H[k]$ can be calculated from (10) as:

$$
H[k]=Y_{p}[k] / X_{p}[k] .
$$

The CSS are demodulated next, obtaining $Y_{C S S}[k]$, and the equalized CSS symbols $X^{e q}[k]$ are calculated as follows:

$$
X^{e q}[k]=\frac{Y_{C S S}[k]}{H[k]},
$$

where it has been assumed that the channel response remains constant between the pilot and the CSS symbols. After equalization, the $X^{e q}[k]$ symbols are demapped into $X_{i}^{e q}[n]$, where $i=1, \ldots, M$, and $n=1, \ldots, L$, obtaining the received $M$ CSS of length $L$. The binary sample $n$ from sequence $i$ is recovered after bit decision:

$$
S_{i}[n]=\left\{\begin{array}{r}
1, \text { if } X_{i}^{e q}[n]>0 \\
-1, \text { if } X_{i}^{e q}[n]<0
\end{array}\right.
$$

Once the $M$-CSS of length $L$ are recovered after demodulation, the correlations are performed in baseband against the original sequences in $Q$ branches, where in this work $Q=2$ : one for the reference beacon, and one for the others. By performing the correlation in baseband, the ideal properties of the CSS are kept, as opposed to correlations with the modulated signal. A CSS correlation peak of amplitude $A_{p}$ is validated if the normalized amplitude $\phi$ satisfies:

$$
\phi=\frac{A_{p}}{M \cdot L} \geq 0.5 \text {. }
$$

By comparing all the normalized peak amplitudes from the $Q$ branches, the detector is able then to validate those candidate arrivals that meet the condition given in (14), rejecting outliers at the same time that identifies the beacon based on the branch with maximum value. After peak validation, TDOAs are computed with reference to B1, and they are transformed to distances by using a nominal sound speed value of $343 \mathrm{~m} / \mathrm{s}$. These distances are then fed to a Gauss-Newton algorithm which calculates the $2 \mathrm{D}$ position.

\section{EXPERIMENTAL Results}

This section describes a series of experiments to test the performance of the proposed system. Three different tests have been conducted: 1) a characterization of the localization accuracy under static conditions, 2) an evaluation of the localization accuracy under motion, and 3) the localization of a mobile robot following different trajectories in the LPS area. Next, the results obtained for the different experiments are presented, and their most relevant aspects are discussed.

\section{A. System Characterization}

First, a system characterization to check the accuracy at rest in the entire positioning area is carried out. For this purpose, a 49-point test grid with a spacing of $0.4 \mathrm{~m}$ has been arranged with the help of a laser rangefinder. A hundred positioning measurements are taken for each test point by placing the microphone at $0.755 \mathrm{~m}$ height. Subsequently, the percentage of valid measurements for each test point (availability), and the mean error for those valid measurements are evaluated. In Fig. 4 it can be observed the results obtained in the experiment. At each of these test points, the mean positioning error in meters is displayed numerically, and the system availability percentage is indicated according to a color scale.

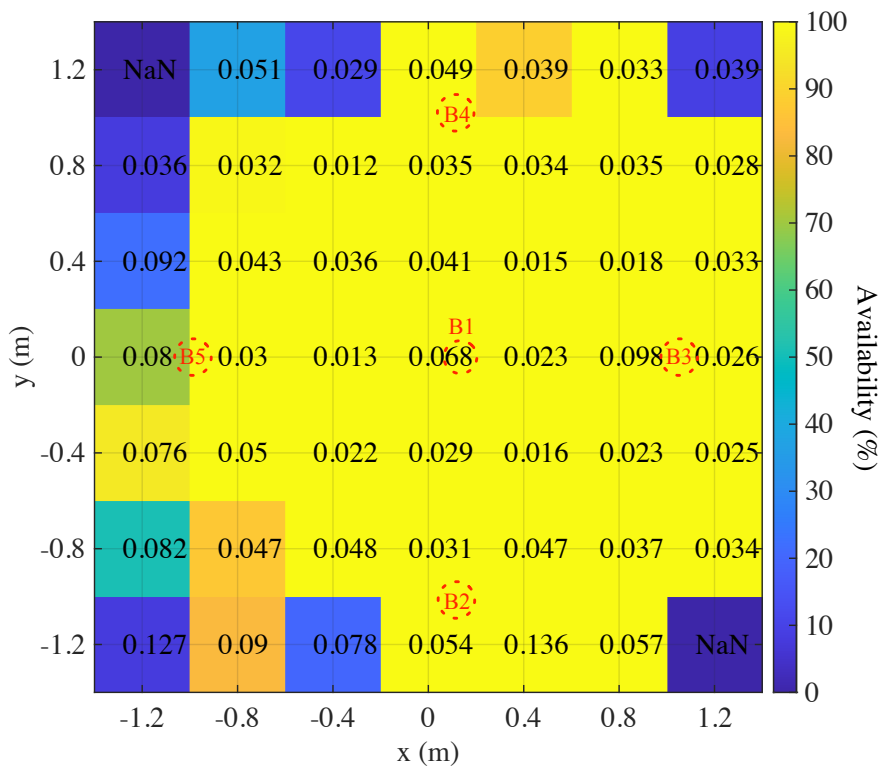

Fig. 4: Mean error (m) and system availability (\%) at rest for the entire coverage area. The dashed red circles mark the beacons projection on the floor.

In Fig. 4 can be appreciated how the system availability is $100 \%$ in a large region of the positioning area. However, it can also be seen how in the peripheral areas the system availability deteriorates, even becoming null in the corners, and the mean error increases. This later effect is caused by the Geometric Dilution of Precision (GDOP) generated by the spatial distribution of the beacons. Although this phenomenon has been minimized by the LPS's geometry, it is still having a certain influence that is accentuated in the peripheral regions.

The main reason behind the loss of availability in these peripheral regions is multipath. Even if the CSS code was designed with a cyclic prefix of $4 \mathrm{~ms}$, thus being resilient against multipath from nearby walls, the received data in peripheral regions showed an additional path with a delay of $16 \mathrm{~ms}$ for the far-away beacons. For example, in the upper-left corner in Fig. 4, this multipath was particularly noticeable in the slots corresponding to the signals transmitted from B2 and B3. This delay is consistent with a floor-ceiling rebound from the transmitted mainlobe, whereas the main path came predominantly from a sidelobe, which had smaller amplitude. Both paths had then comparable energy, and the multipath introduced errors in the Doppler estimation, and could potentially cause erroneous demodulation of the CSS code, resulting in beacons not being validated. If more than two beacons are lost, then no position can be calculated by the system. Additionally, if the beacon B1 is lost, then no position is obtained by the system, since there is no reference to calculate the TDOA. This last situation happened in $17 \%$ of the measures in the point $(-1.2,0.8)$, and $18 \%$ in $(-1.2,0)$, for 
example. Also, if a multipath signal is validated instead of the correct peak, the Gauss-Newton algorithm would not converge. Finally, the Signal to Noise Ratio (SNR) degradation of validated signals in low coverage areas must also be considered. In this experiment, these SNR values range from a maximum of $22.4 \mathrm{~dB}$ to a minimum of $2.6 \mathrm{~dB}$. All these effects combined reduced the availability of the system in the peripheral regions.

\section{B. Positioning Accuracy in Motion}

The objective of this test is to evaluate the behavior of the detector under strong Doppler shifts. The experimental setup includes: a 2-meter long electric actuator [47] powered by a $24 \mathrm{~V}$ DC source, an acquisition board [48], an ultrasonic microphone GRAS 40BE 1/4 [41] with its amplifying signal unit [49] and a laptop in which a Labview ${ }^{\circledR}$ program is executed. This program is in charge of synchronizing the electric actuator movement and the received signal acquisition. Once the movement ends, the acquired data are sent to the laptop to be processed with Matlab ${ }^{\circledR}$. This assembly is shown in Fig. 5.

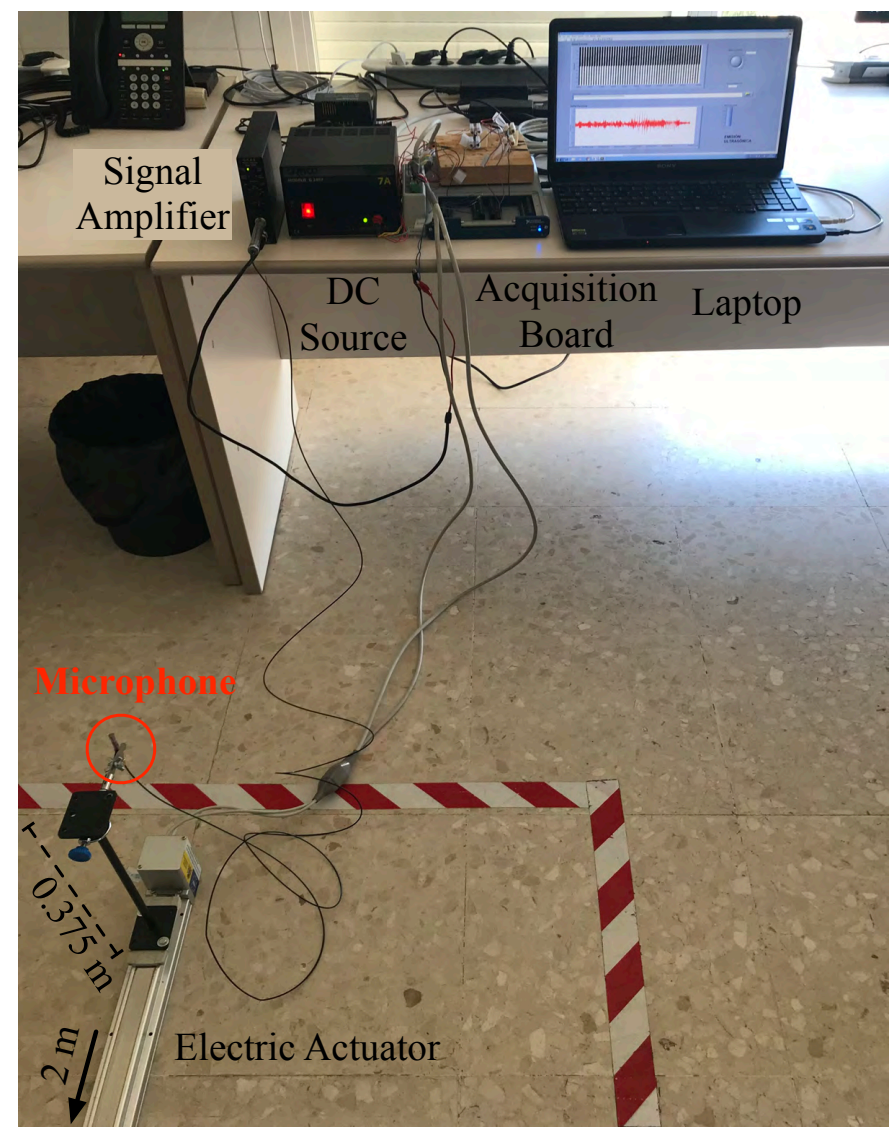

Fig. 5: Electric actuator positioning setup.

The assembly described in Fig. 5 is used to establish a ground truth reference to evaluate the positioning accuracy in motion. In this way, knowing the rectilinear motion equation described by the electric actuator, it is possible to calculate the theoretical location points reached by the receiver in the instants defined by B1's TOAs.
The actuator is located on the $x$-axis $(y=0)$, making the midpoint of the distance traveled coincide with the $y$ axis $(x=0)$. Experimental tests are performed for maximum electric actuator speeds of $0.5,1.0,1.5$ and $2.0 \mathrm{~m} / \mathrm{s}$. These speeds are reached at $3 \mathrm{~m} / \mathrm{s}^{2}$, the device's maximum allowed acceleration. Therefore, actuator starts at $x=1 \mathrm{~m}$ accelerating to $3 \mathrm{~m} / \mathrm{s}^{2}$ until it reaches the established maximum speed, remains at this maximum speed for some time and then decelerates to $-3 \mathrm{~m} / \mathrm{s}^{2}$ until rest at $x=-0.8 \mathrm{~m}$. Since the acquisition of the signal is synchronized with the rectilinear movement of the electric actuator, the theoretical ground truth points can be easily obtained by substituting the detection instants of B1 (reference beacon) in the equation of the trajectory.

Fig. 6 shows the Doppler shift measured from the received signal from B1 at the different ground truth positions. After the movement starts at $x=1 \mathrm{~m}$, the Doppler shifts changes non linearly due to the effect of the actuator acceleration. Then the measured Doppler shift changes linearly as the receiver moves with constant speed, and it becomes negative after passing below B1, moving away from it. It can be also noticed how the magnitude of the Doppler shift depends on the actuator speed.

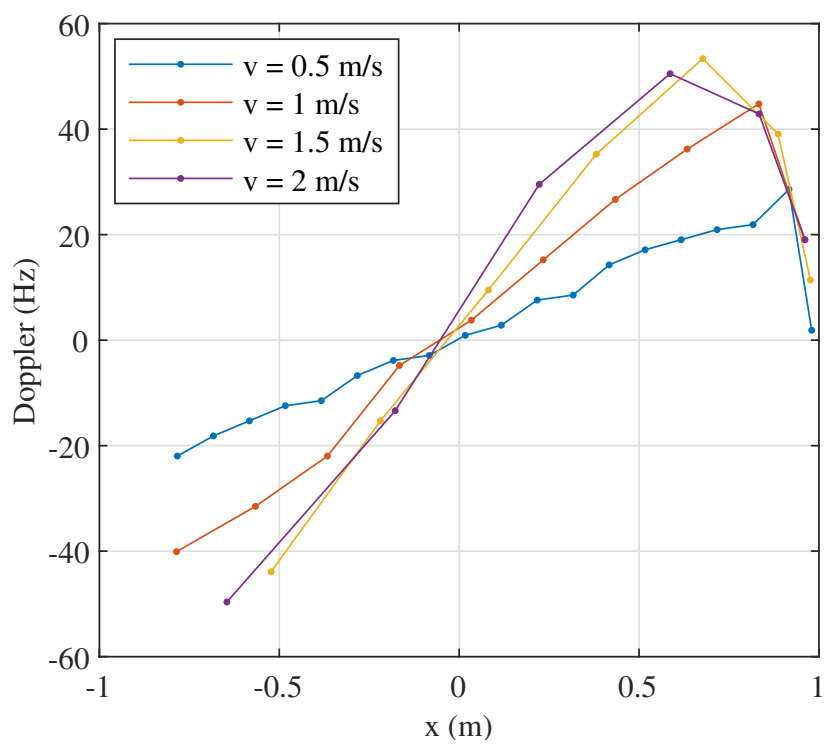

Fig. 6: Doppler shifts measured from B1's received signal, for different electric actuator speeds.

In Fig. 7, a comparison between the measured positions and the ground truth for each speed can be observed, where only the positions obtained while the receiver is moving have been plotted. As shown in this figure, the best positioning results for all speeds are obtained in the central region of the trajectory. This zone corresponds to the time in which the actuator moves at a constant speed, and therefore the received signal is not affected by acceleration. In this central region (according to Fig. 4), good system GDOP and SNRs above $12.6 \mathrm{~dB}$ also contribute to these results. In contrast, in the initial and final trajectory parts, the positioning accuracy and SNRs, around 2.1 dB, worsen. In the initial part, the main cause of the 
accuracy decrease is the acceleration. However, in the final part of the trajectory, both a small friction in the actuator's movement, not contemplated in the ground truth estimation, and the deceleration effect, have a remarkable influence on the obtained positioning accuracy.

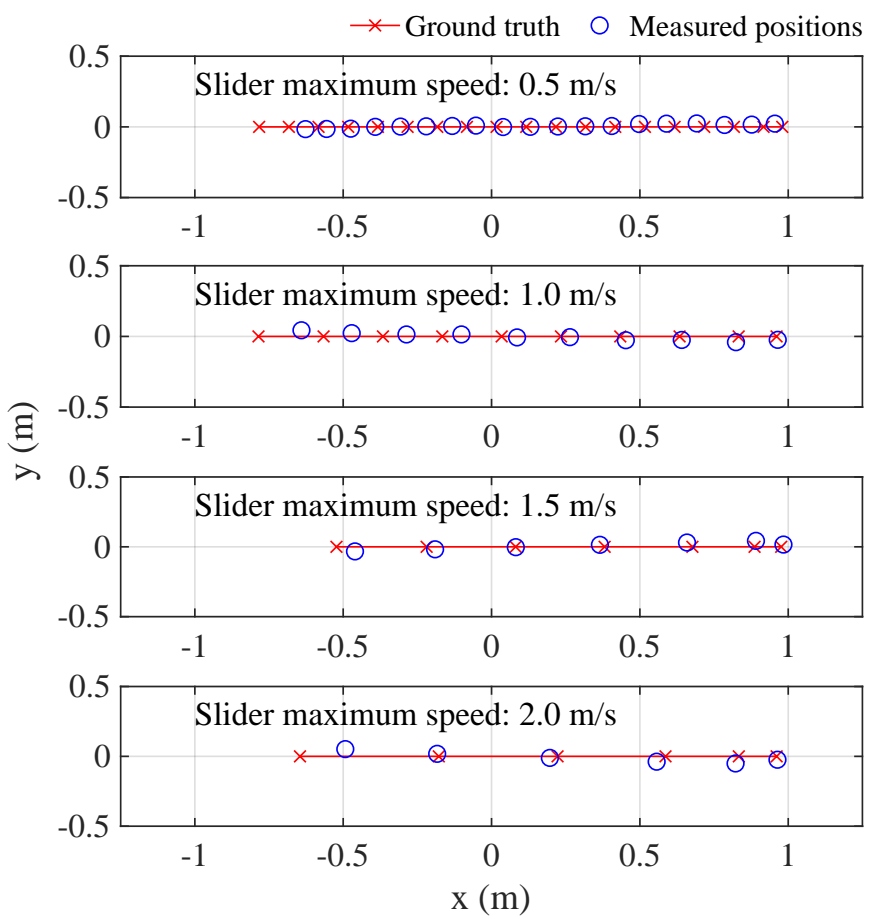

Fig. 7: Positioning results at different electric actuator speeds.

Detailed in the TABLE I is the number of test points taken, together with the minimum, maximum and mean positioning errors obtained for each maximum slider speed case. As can be seen in this table, the mean positioning errors are below $6 \mathrm{~cm}$ in any case. Also, the maximum errors in the most adverse circumstances do not exceed $16.1 \mathrm{~cm}$.

TABLE I: Positioning errors obtained for different slider maximum speeds.

\begin{tabular}{|c|c|c|c|c|c|}
\hline Slider Maximum & Trajectory & Number of & \multicolumn{3}{|c|}{ Positioning Error $(\mathrm{m})$} \\
\cline { 4 - 6 } Speed $(\mathrm{m} / \mathrm{s})$ & Time $(\mathrm{s})$ & Test Points & Min & Max & Mean \\
\hline 0.5 & 3.767 & 19 & 0.004 & 0.157 & 0.051 \\
\hline 1.0 & 2.133 & 10 & 0.024 & 0.151 & 0.060 \\
\hline 1.5 & 1.700 & 7 & 0.027 & 0.070 & 0.032 \\
\hline 2.0 & 1.567 & 6 & 0.019 & 0.161 & 0.055 \\
\hline
\end{tabular}

\section{Mobile Robot Positioning}

In this assembly, a mobile acquisition system placed on a Pioneer ${ }^{\circledR}$ P3-DX robot [50] is used. This system consists of: an Avisoft ${ }^{\circledR}$ acquisition module [51], a conditioning unit [52], an ultrasonic microphone GRAS 40BE 1/4 [41] and a laptop where the acquisition software is run. In this case, both signal acquisition and robot motion start at different times. This setup is detailed in Fig. 8.

To perform this test, the robot is situated in the center of the positioning area and is programmed to perform a square

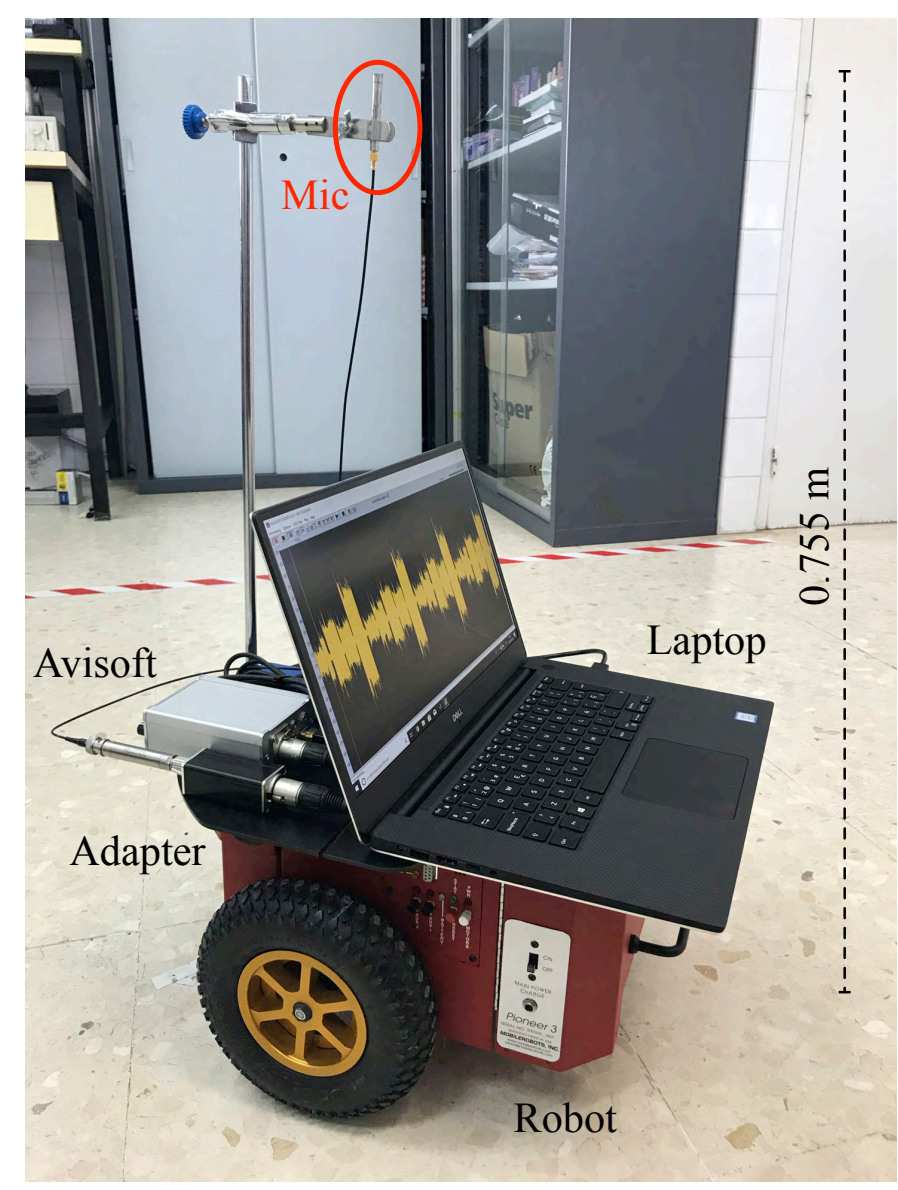

Fig. 8: Mobile robot positioning setup.

trajectory around the origin at a maximum speed of $1.2 \mathrm{~m} / \mathrm{s}$. In total, three different trajectories are programmed, where squares of 1.0, 1.5 and 2.0 meters of side are defined. For each of these trajectories, the received signal is saved and processed offline with Matlab ${ }^{\circledR}$ to obtained its positioning results.

From the positioning results shown in Fig. 9, it can be inferred a slight rotation in the trajectories described by the mobile robot. This circumstance is due to the low ground adhesion causing the wheels to skid when the robot rotates on itself and therefore, introducing a small error in the robot odometry system. Therefore, it is impossible to use odometry as a reliable system to establish a comparative ground truth.

It can be observed how in the 1-meter-side trajectory the positioning is quite robust due to the good cover conditions obtaining SNR values ranges from a minimum of $5.6 \mathrm{~dB}$ to a maximum of $20.3 \mathrm{~dB}$. The location of the robot was estimated throughout the whole trajectory, which consisted of 182 points. Blue circles represent those positioning points where beacons were validated. Only at one point in the upper-left corner the receiver did not validate one of the beacons. In that case, the position was estimated using four beacons. On the other hand, the red crosses indicate positioning points obtained by means of a classical TDMA architecture, i.e, the correct peak location of B1 is fed to the classical system, which then looks for the other chirps from the remaining beacons, knowing their corresponding time search windows. In this case, the position 




Fig. 9: Positioning result for different robot trajectories.

has been lost in 3 points of the trajectory.

The 1.5-meters-side trajectory consists of 213 points where the SNR ranges from $2.2 \mathrm{~dB}$ to $21.2 \mathrm{~dB}$. Green circles represent positioning points using the proposed beacon validation method. With the two-step validation method, the position of the robot was estimated in 209 of them $(98.12 \%)$, of which 176 points $(82.62 \%)$ were positioned by validating 5 beacons, 31 points $(14.55 \%)$ with 4 beacons, and 2 points $(0.93 \%)$ through the validation of only 3 beacons. However, positioning in 4 points $(1.87 \%)$ was not possible, in three of them due to the loss of the reference beacon and in one due to the nonconvergence of the Gauss-Newton algorithm. The positions calculated by the classical system are again represented by red crosses. In this case, the position was achieved in 155 points from the 210 with a valid reference $(73.80 \%)$.

As expected based on the results from Fig. 4, the performance decreased in the 2-meter-side trajectory. In this trajectory, SNR values extend from $4.5 \mathrm{~dB}$ to $22.2 \mathrm{~dB}$. The positions when beacons were validated are represented by yellow circles, whereas red crosses represent again the positions obtained by the classical system. It can be drawn from the figure that the upper and left parts of the trajectory are especially challenging for the positioning system. Fig. 10 shows the measured Doppler shift for the beacons that experienced less Doppler variation (B1, in blue), and more variation (B3, in red). Different sections can be noticed where the robot came closer or separated from the beacons, as it followed the predetermined trajectory. Several points are missing for B3 between frames 125 and 175 approximately, which corresponds to the upper-left corner of the trajectory where B3 could not be validated. For B3, the measured Doppler shift ranged around $100 \mathrm{~Hz}$.

An example of a typical reception in the upper-right corner of the peripheral region is shown in Fig. 11. It shows the

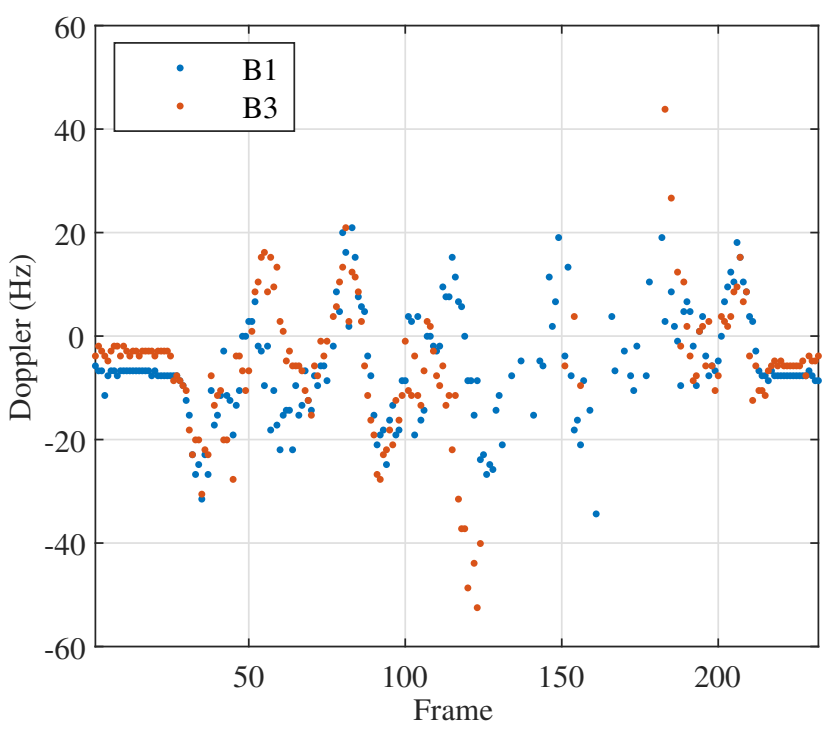

Fig. 10: Doppler shifts measured at B1 and B3, for the entire trajectory.

correlation signal with the chirp, where all the candidate peaks are marked with black crosses. It can be seen how the signals from B2 and B5 are more attenuated compared to the other three, as expected. They also exhibit the aforementioned floor-ceiling multipath, with a comparable energy than the main path. The two-step detection and validation method goes through all candidates, and identifies B1 as the reference beacon, and also B3 and B4, all marked with red circles. The constellation of the received (Rx) demodulated QPSK symbols for B1's code is shown in Fig. 12, together with the correlation with the original CSS code, in case of applying Doppler compensation (upper subfigures), and ignoring the Doppler compensation step (bottom subfigures). If no Doppler compensation is applied, more symbols are incorrectly demodulated, obtaining a smaller value for the normalized correlation peak. In this particular case, this peak could have still been validated, but if the peak had gone under the threshold of 0.5 , that arrival would not have been validated. If less than 3 beacons are validated, the location in that frame cannot be obtained.

Since in this case it validates three beacons, it is able to calculate a 2D location at $(0.8939,0.9783) \mathrm{m}$. However, the classical system has no way to verify the validity of the peaks, so it always needs to provide five peaks to the localization algorithm, one for each beacon, assuming they are correct. Detections from the classical method are shown with yellow squares. It correctly identified B3 and B4, but it also validated multipath peaks from B1 and B2, erroneously identifying the beacons and causing the Gauss-Newton algorithm to diverge. As a result, no position was obtained using the classical system. This problem originated as the multipath signal from B1 and B2 had higher amplitudes than the main path from B2 in those slots. As multipath and attenuation from faraway beacons is more predominant in larger trajectories, the classical system decreases its availability as correct identifi- 


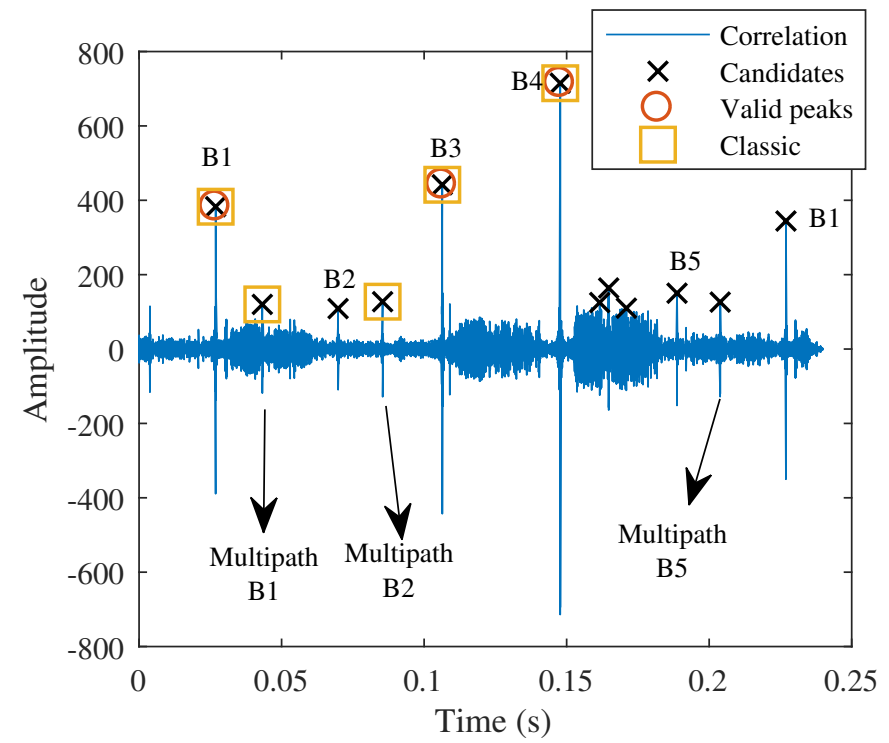

Fig. 11: Identification of peaks in a $240 \mathrm{~ms}$ frame, using the classical system (yellow squares) and the two-step detection method (red circles).

cation becomes more challenging. The position of the robot was calculated in 200 out of 232 points $(86.20 \%)$ using the proposed two-step validation method. Of these, 150 points $(64.65 \%)$ were positioned validating 5 beacons, 43 points $(18.53 \%)$ validating 4 beacons and 7 points (3.01\%) validating 3 beacons. The reasons for not obtaining a valid position in the remaining points were: 1) losing the reference beacon in 26 points (11.21\%), and 2) non-convergence of the GaussNewton algorithm in 6 points (2.59\%). Considering the 206 points with a valid reference, positioning was achieved by the classical system in 136 of them (66.01\%).

\section{CONCLUSIONS}

In this work, a two-step detection and validation method has been presented for an ultrasonic LPS, based on the use of CSS as a second step validation code. The key of this second step is the use of OFDM modulated CSS signals. The combination of easy channel equalization provided by the OFDM modulation, together with the ideal auto- and crosscorrelation properties of CSS when performed in baseband, allow to design a simple peak detector for this second step. The combination of this validation step with the chirp detection and Doppler compensation provided by the carrier signal, results in a robust method to discern between valid and spurious arrivals, which are known to reduce the availability and accuracy of positioning systems. Signal processing is carried out in acquired data frames of $240 \mathrm{~ms}$, with an overlap of $40 \mathrm{~ms}$. This leads to a theoretical update rate of $5 \mathrm{~Hz}$ that should be enough to guide a mobile robot on a predefined path, as far as the speed of this robot is not too high.

The proposed two-step validation method has been tested in different experiments, in order to assess the performance under different conditions. In static experiments, this method provided a mean error of $4.6 \mathrm{~cm}$, and a mean availability (a) Rx CSS, compensated

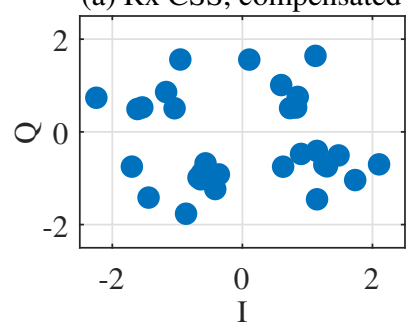

(c) Rx CSS, uncompensated
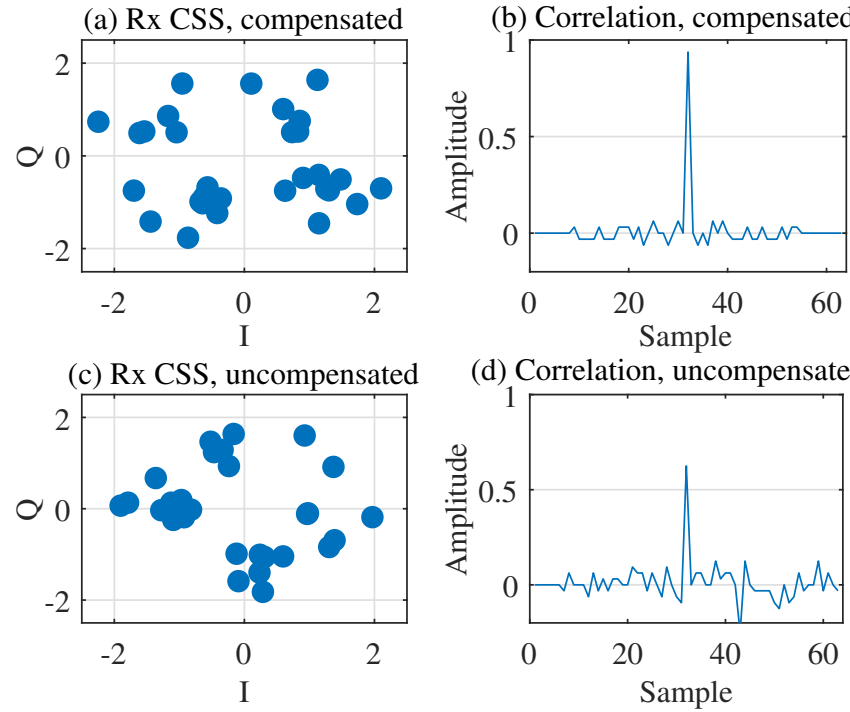

(d) Correlation, uncompensated

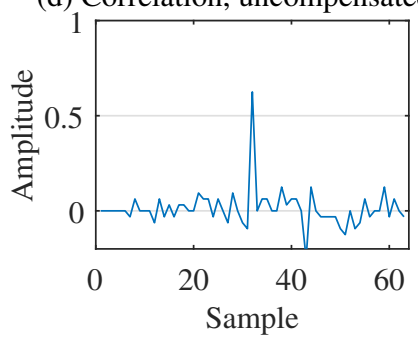

Fig. 12: Demodulated CSS symbols (a) and correlation (b) considering Doppler compensation, and CSS symbols (c) and correlation (d) without compensation.

of $81.4 \%$. Under high speeds and strong Doppler effects, it provided positions with a mean error of $6 \mathrm{~cm}$ for the worst case. And finally, in order to assess the benefits of this twostep validation method with regard to a classical detection method, a comparison has been performed for three different square trajectories of $1,1.5$ and $2 \mathrm{~m}$ sides. The two-step method increased the robustness of the LPS in all cases, providing availabilities of $100 \%, 98.1 \%$ and $86.2 \%$, for those three trajectories. The improvement can be specially noticed in the 2-meter-side trajectory, where multipath, near-far effect and low amplitude signals are predominant, and the classical method had more difficulties selecting the correct peaks. In this case, the availability of the system increased from $66.01 \%$ to $86.20 \%$, by using the two-step validation process. The effect of this robustness and performance improvement is an increase of the coverage area of the LPS.

In future work, additional changes in the design will be explored in order to being able to dynamically change the reference beacon, from a fixed one to the valid arrival with highest energy in a frame, as well as exploring the benefits of using a longer cyclic prefix to deal with late multipath arrivals. By doing this, it would be possible to further increase the availability of the system, since losing the reference beacon and multipath are the main cause of non-availability.

\section{ACKNOWLEDGMENT}

The authors would like to thank Prof. Sverre Holm and Forkbeard Technologies AS for their support.

\section{REFERENCES}

[1] S. Steiniger, M. Neun, and A. Edwardes, "Foundations of location based services," Project CartouCHe1-Lecture Notes on LBS V 1.0, Dept. of Geography, University of Zurich, 2006.

[2] R. Mautz, "Indoor positioning technologies," Habilitation Thesis, Dept. of Civil, Environmental and Geomatic Engineering, ETH Zurich, 2012. 
[3] F. Zafari, A. Gkelias, and K. K. Leung, "A survey of indoor localization systems and technologies," IEEE Commun. Surv. Tut., vol. 21, no. 3, pp. 2568-2599, Apr. 2019.

[4] F. J. Álvarez, "Fundamentals of airborne acoustic positioning systems," in Geographical and Fingerprinting Data to Create Systems for Indoor Positioning and Indoor/Outdoor Navigation, J. Conesa, A. Pérez, J. Torres, and R. Montoliu, Eds. Cambridge, MA, USA: Elsevier AP, 2018, ch. 17 , pp. $335-352$.

[5] Forkbeard Technologies AS, last accessed on February 25th, 2020. [Online]. Available: https://forkbeardtech.com

[6] M. Benyamin and G. H. Goldman, "Acoustic detection and tracking of a Class I UAS with a small tetrahedral microphone array," Army Research Lab Adelphi MD, Tech. Rep., 2014.

[7] J. Büsset, F. Perrodin, B. Ott, K. Heutschi, T. Rühl, and T. Nussbaumer, "Detection and tracking of drones using advanced acoustic cameras," in Proc. SPIE, vol. 9647, Toulouse, France, 2015, pp. 9647-9647.

[8] J. A. Paredes, F. J. Álvarez, T. Aguilera, and J. M. Villadangos, “3D indoor positioning of UAVs with spread spectrum ultrasound and timeof-flight cameras," Sensors-Basel, vol. 18, no. 1, article no. 89, Jan. 2018.

[9] M. Alloulah and M. Hazas, "An efficient CDMA core for indoor acoustic position sensing," in Proc. IEEE IPIN, Zurich, Switzerland, 2010, pp. $1-5$

[10] J. A. Paredes, T. Aguilera, F. J. Álvarez, J. Lozano, and J. Morera, "Analysis of Doppler effect on the pulse compression of different codes emitted by an ultrasonic LPS," Sensors-Basel, vol. 11, no. 11, pp. 10765-10 784, Nov. 2011.

[11] F. J. Álvarez, A. Hernández, J. A. Moreno, M. C. Pérez, J. Ureña, and C. De Marziani, "Doppler-tolerant receiver for an ultrasonic LPS based on Kasami sequences," Sensor. Actuat. A-Phys., vol. 189, pp. 238-253, Jan. 2013.

[12] S. Widodo, T. Shiigi, N. Hayashi, H. Kikuchi, K. Yanagida, Y. Nakatsuchi, Y. Ogawa, and N. Kondo, "Moving object localization using sound-based positioning system with Doppler shift compensation," Robotics, vol. 2, no. 2, pp. 36-53, Apr. 2013.

[13] X. Chen, Y. Chen, S. Cao, L. Zhang, X. Zhang, and X. Chen, "Acoustic indoor localization system integrating TDMA+FDMA transmission scheme and positioning correction technique," Sensors-Basel, vol. 19, no. 10, article no. 2353, May 2019.

[14] T. Aguilera, F. J. Álvarez, J. A. Paredes, and J. A. Moreno, "Doppler compensation algorithm for chirp-based acoustic local positioning systems," Digit. Signal Process., 2020, accepted, to be published.

[15] J. M. Villadangos, J. Ureña, M. Mazo, A. Hernández, C. De Marziani, A. Jiménez, and F. J. Álvarez, "Improvement of cover area in ultrasonic local positioning system using cylindrical PVDF transducer," in Proc. IEEE ISIE, Vigo, Spain, 2007, pp. 1473-1477.

[16] D. Gualda, J. Ureña, J. J. García, A. Jiménez, M. C. Pérez, and F. Ciudad, "Coverage analysis of an ultrasonic local positioning system according to the angle of inclination of the beacons structure," in Proc. IEEE IPIN Pisa, Italy, 2019, pp. 1-7.

[17] D. T. Blackstock, Fundamentals of Physical Acoustics. Hoboken, NJ, USA: John Wiley \& Sons, 2000.

[18] ISO/TC 43 Technical Comittee, Acoustics, Sub-Comittee SC1, Noise, "Attenuation of sound during propagation outdoors. Part 1: Calculation of the absorption of sound by the atmosphere," International Organization for Standarization, Tech. Rep. ISO 9613-1:1993(E), 1993.

[19] A. Ward, A. Jones, and A. Hopper, "A new location technique for the active office," IEEE Pers. Commun., vol. 4, no. 5, pp. 42-47, Oct. 1997.

[20] E. Foxlin and M. Harrington, "Constellation: a wide-range wireless motion-tracking system for augmented reality and virtual set applications," in Proc. ACM SIGGRAPH, Orlando, FL, USA, 1998, pp. 371378.

[21] N. B. Priyantha, A. Chakraborty, and H. Balakrishnan, "The Cricket location-support system," in Proc. ACM MobiCom, Boston, MA, USA, 2000, pp. 32-43.

[22] M. I. Skolnik, Introduction to Radar Systems, 2nd ed. New York, NY, USA: McGraw-Hill Book Co., 1980.

[23] H. Peremans, K. Audenaert, and J. M. Van Campenhout, "A highresolution sensor based on tri-aural perception," IEEE T. Robotic. Autom., vol. 9, no. 1, pp. 36-48, Feb. 1993.

[24] K.-W. Jörg and M. Berg, "Sophisticated mobile robot sonar sensing with pseudo-random codes," Robot. Auton. Syst., vol. 25, no. 3, pp. 241-251, Nov. 1998.

[25] J. Ureña, M. Mazo, J. J. García, A. Hernández, and E. Bueno, "Classification of reflectors with an ultrasonic sensor for mobile robot applications," Robot. Auton. Syst., vol. 29, no. 4, pp. 269-279, Dec. 1999.
[26] M. Hazas and A. Ward, "A novel broadband ultrasonic location system," in Proc. ACM UbiComp, Göteborg, Sweden, 2002, pp. 264-280.

[27] _ "A high performance privacy-oriented location system," in Proc. IEEE PerCom, Fort Worth, TX, USA, 2003, pp. 216-223.

[28] J. M. Villadangos, J. Ureña, M. Mazo, A. Hernández, F. J. Álvarez, J. J. García, C. De Marziani, and D. Alonso, "Improvement of ultrasonic beacon-based local position system using multi-access techniques," in Proc. IEEE WISP, Faro, Portugal, 2005, pp. 352-357.

[29] J. Ureña, A. Hernández, A. Jiménez, J. M. Villadangos, M. Mazo, J. C. García, J. J. García, F. J. Álvarez, C. De Marziani, M. C. Pérez, J. A. Jiménez, A. R. Jiménez, and F. Seco, "Advanced sensorial system for an acoustic LPS," Microproces. Microsy., vol. 31, no. 6, pp. 393-401, Sept. 2007.

[30] M. C. Pérez, J. Ureña, A. Hernández, C. De Marziani, A. Jiménez, J. M. Villadangos, and F. J. Álvarez, "Ultrasonic beacon-based local positioning system using Loosely Synchronous codes," in Proc. IEEE WISP, Alcalá de Henares, Spain, 2007, pp. 1-6.

[31] M. C. Pérez, R. S. Serrano, J. Ureña, A. Hernández, C. De Marziani, and F. J. Álvarez, "Correlator implementation for orthogonal CSS used in an ultrasonic LPS," IEEE Sens. J., vol. 12, no. 9, pp. 2807-2816, Sept. 2012.

[32] S. Murano, M. C. Pérez, D. Gualda, F. J. Álvarez, T. Aguilera, and C. De Marziani, "Evaluation of Zadoff-Chu, Kasami and chirp based encoding schemes for acoutic local positioning systems," IEEE T. Instrum. Meas., vol. Early Access, pp. 1-13, 2020.

[33] D. F. Albuquerque, J. M. Vieira, S. I. Lopes, C. A. Bastos, and P. J. Ferreira, "OFDM pulse design with low PAPR for ultrasonic location and positioning systems," in Proc. IEEE IPIN, Montbeliard, France, 2013, pp. 34-35.

[34] F. J. Álvarez, T. Aguilera, and R. López-Valcarce, "CDMA-based acoustic local positioning system for portable devices with multipath cancellation," Digit. Signal Process., vol. 62, no. Supplement C, pp. 38-51, Mar. 2017.

[35] C. De Marziani, J. Ureña, Á. Hernández, J. J. García, F. J. Álvarez, A. Jiménez, M. C. Pérez, J. M. Villadangos, J. Aparicio, and R. Alcoleas, "Simultaneous round-trip time-of-flight measurements with encoded acoustic signals," IEEE Sens. J., vol. 12, no. 10, pp. 2931-2940, Jun. 2012.

[36] D. F. Albuquerque, J. M. N. Vieira, S. I. Lopes, T. Aguilera, and F. J. Álvarez, "Doppler resilient modulation in a CDMA-based acoustic local positioning system," in Proc. IEEE IPIN, Alcalá de Henares, Spain, 2016, pp. $1-8$.

[37] NXP LPC1768, "ARM microcontroller," last accessed on February 25th, 2020. [Online]. Available: https://www.nxp.com/docs/en/datasheet/LPC1769_68_67_66_65_64_63.pdf

[38] Prowave 328ST/R160, "Air ultrasonic ceramic transducers," last accessed on February 25th, 2020. [Online]. Available: http://www. prowave.com.tw/english/products/ut/open-type/328s 160 .htm

[39] R. B. Langley, "Dilution of precision," GPS world, vol. 10, no. 5, pp. 52-59, May 1999.

[40] Bosch GLM 80, "Laser rangefinder," last accessed on February 25th, 2020. [Online]. Available: http://www.bosch-professional.com/sa/ en/laser-measure-glm-80-131500-0601072370.html

[41] GRAS 40BE 1/4, "Ultrasonic microphone," last accessed on February 25th, 2020. [Online]. Available: https://www.gras.dk/products/measurement-microphone-cartridge/ prepolarized-cartridges-0-volt/product/158-40be

[42] J. Aparicio and T. Shimura, "Asynchronous detection and identification of multiple users by multi-carrier modulated complementary set of sequences," IEEE Access, vol. 6, pp. 22 054-22 069, Apr. 2018.

[43] J. Aparicio, "Evaluation of spread-spectrum signals in realistic underwater noisy environments," in Proc. IEEE/MTS OCEANS, Shanghai, China, 2016, pp. 1-9.

[44] C. Tseng and C. L. Liu, "Complementary sets of sequences," IEEE T. Inform. Theory, vol. IT-18, no. 5, pp. 644-652, Sept. 1972.

[45] J. A. C. Bingham, "Multicarrier modulation for data transmission: An idea whose time has come," IEEE Commun. Mag., vol. 28, no. 5, pp. 5-14, May 1990.

[46] N. Yoder, "Peakfinder," last accessed on February 25th, 2020. [Online]. Available: https://www.mathworks.com/matlabcentral/fileexchange/ 25500-peakfinder-x0--sel--thresh--extrema--includeendpoints-interpolate-

[47] SMC LEFB25AT-2000, "Electric actuator," last accessed on February 25th, 2020. [Online]. Available: https://www.smcpneumatics.com/ LEFB25AT-2000.html 
[48] NI USB-6363, "Multifunction I/O device," last accessed on February 25th, 2020. [Online]. Available: http://www.ni.com/en-gb/support/ model.usb-6363.html

[49] GRAS 12AK 1, "Channel power module," last accessed on February 25th, 2020. [Online]. Available: https://www.gras.dk/products/powermodule/product/225-12ak

[50] Pioneer P3-DX, "Mobile robot," last accessed on February 25th, 2020. [Online]. Available: https://www.generationrobots.com/media/ Pioneer3DX-P3DX-RevA.pdf

[51] Avisoft UltraSoundGate $116 \mathrm{H}$ basic, "Recording interface," last accessed on February 25th, 2020. [Online]. Available: http://www. avisoft.com/ultrasoundgate/116hb/

[52] Avisoft UltraSoundGate 1/4" , "Mic power module," last accessed on February 25th, 2020. [Online]. Available: http://www.avisoft.com/ ultrasound-microphones/1-4-mic-power-module/

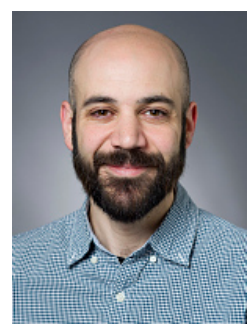

Joaquín Aparicio (M'17) received his physics degree from the University of Extremadura, Badajoz, Spain, in 2008, and his Ph.D. degree in electronics from the University of Alcalá, Alcalá de Henares, Spain, in 2014.

From 2015 to 2018 he was a Postdoctoral Researcher at the Japan Agency for MarineEarth Science and Technology (JAMSTEC), Yokosuka, Japan. Since 2018 he is a Postdoctoral Fellow at the Department of Informatics, University of Oslo, Oslo, Norway. His research interests include positioning systems, acoustic propagation and signal processing.

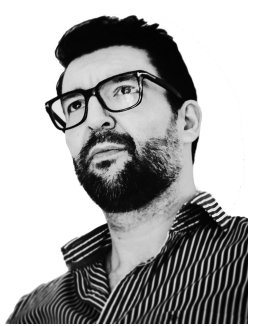

Teodoro Aguilera received his physics degree in 2009 and his PhD. in electronics in 2016 all of them from the University of Extremadura, Badajoz, Spain.

He was an Assistant Professor of Automation for three years in the Department of Electrical Engineering, Electronics and Automation at the University of Extremadura. Currently he is a research member of the Sensory Systems Group of this university where his work lies in the design of Acoustic Local Positioning Systems (ALPS) based on mobile devices.

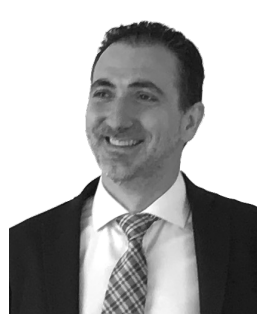

Fernando J. Álvarez (M'07 - SM'17) received his physics degree from the University of Sevilla, Sevilla, Spain, his electronic engineering degree from the University of Extremadura, Badajoz, Spain, and his Ph.D. degree in electronics from the University of Alcalá, Alcalá de Henares, Spain. He also holds a M.Sc. degree in signal theory and communications from the University of Vigo, Vigo, Spain, and a M.Sc. degree in telecommunications engineering from the Universitat Oberta de Catalunya, Barcelona, Spain.

Since 2001, he has been with the Department of Electrical Engineering, Electronics and Automation at the University of Extremadura, where he is currently a Full Professor and Head of the Sensory Systems Research Group. In 2008, he joined the Intelligent Sensors Laboratory, Yale University, New Haven, CT, USA, as a Post-Doctoral "José Castillejo" Fellow. His current research interests include local positioning systems, acoustic signal processing, and embedded computing. 